\title{
Predictors of Overall and Disease-Free Survival in Metastatic Castration-Resistant Prostate Cancer Patients Receiving ${ }^{225}$ Ac-PSMA-617 Radioligand Therapy
}

\author{
Mike Sathekge ${ }^{1}$, Frank Bruchertseifer ${ }^{2}$, Mariza Vorster $^{1}$, Ismaheel O. Lawal ${ }^{1}$, Otto Knoesen ${ }^{3}$, Johncy Mahapane ${ }^{1}$, \\ Cindy Davis ${ }^{1}$, Florette Reyneke ${ }^{1}$, Alex Maes ${ }^{1,4}$, Clemens Kratochwil ${ }^{5}$, Thabo Lengana ${ }^{1}$, Frederik L. Giesel ${ }^{5}$, \\ Christophe Van de Wiele ${ }^{1,6}$, and Alfred Morgenstern ${ }^{1,2}$ \\ ${ }^{1}$ Department of Nuclear Medicine, Steve Biko Academic Hospital, University of Pretoria, Pretoria, South Africa; ${ }^{2}$ European Commission, \\ Joint Research Centre, Directorate for Nuclear Safety and Security, Karlsruhe, Germany; ${ }^{3}$ Nuclear Technology Products (NTP), \\ Pelindaba, South Africa; ${ }^{4}$ Katholieke University Leuven, Kortrijk, Belgium; ${ }^{5}$ University Hospital Heidelberg, Heidelberg, Germany; and \\ ${ }^{6}$ Ghent University, Ghent, Belgium
}

\begin{abstract}
Metastatic prostate carcinoma overexpresses prostate-specific membrane antigen (PSMA), making this antigen a suitable target for radioligand therapy of the disease. Here we report on our experience with a series of 73 castration-resistant prostate carcinoma patients treated with ${ }^{225} \mathrm{Ac}-\mathrm{PSMA}-617$, identifying variables predictive for overall survival (OS) and progression-free survival (PFS) after ${ }^{225}$ Ac-PSMA-617 treatment. Methods: ${ }^{225}$ Ac-PSMA-617 was administered to patients who had metastatic castration-resistant prostate carcinoma and who had exhausted available therapy options for their disease. Full blood count, glomerular filtration rate, and liver function test were obtained at baseline and on follow-up for evaluation of toxicity. ${ }^{68} \mathrm{Ga}$-PSMA PET/CT was obtained at baseline, before every treatment cycle, and on follow-up for selection of patients for treatment, to determine the activity of the treatment agent to be administered, and for response assessment. Serial prostatespecific antigen (PSA) was obtained for PSA response assessment. Results: Seventy-three men (mean age, 69 y; range, 45-85 y) with metastatic castration-resistant prostate carcinoma were treated with 210 cycles of ${ }^{225} \mathrm{Ac}-\mathrm{PSMA}-617$. In $70 \%$ of patients, a PSA decline of greater than or equal to $50 \%$ was obtained; $82 \%$ of patients had any PSA decline. In $29 \%$ of patients, all lesions on ${ }^{68} \mathrm{Ga}-$ PSMA PET resolved in response to treatment. During follow-up, 23 patients experienced disease progression, whereas 13 patients died from their disease. The estimated median PFS and OS were $15.2 \mathrm{mo}(95 \% \mathrm{Cl}, 13.1-17.4)$ and $18 \mathrm{mo}(95 \% \mathrm{Cl}, 16.2-19.9)$, respectively. In univariate analyses, factors such as baseline PSA, any PSA decline, PSA decline of greater than or equal to $50 \%$, prior chemotherapy, prior radiation therapy, and baseline hemoglobin level were associated with longer PFS and OS (all $P$ s $<0.05$ ). In multivariate analyses, there was a negative association between prior ${ }^{177}$ Lu-PSMA therapy and PFS, and a positive association between PSA decline of greater or equal to $50 \%$ and PFS. Only a PSA decline of greater than or equal to $50 \%$ remained significantly associated with OS on multivariate analyses. Xerostomia was seen in $85 \%$ of patients but was not severe enough to warrant discontinuing treatment. Anemia was seen in 27 patients; no patients had grade IV bone marrow toxicity. Renal failure of grade III or IV was
\end{abstract}

Received Mar. 28, 2019; revision accepted May 7, 2019.

For correspondence or reprints contact: Mike Sathekge, Department of Nuclear Medicine, Steve Biko Academic Hospital, University of Pretoria, Private Bag X169, Pretoria, 0001, South Africa.

E-mail: mike.sathekge@up.ac.za

Published online May 17, 2019.

COPYRIGHT (C 2020 by the Society of Nuclear Medicine and Molecular Imaging. seen in 5 patients with baseline renal impairment. Conclusion: In this study, a PSA decline of greater than or equal to $50 \%$ after treatment with ${ }^{225}$ Ac-PSMA- 617 was proven by multivariate analyses to be significantly associated with OS and PFS. Furthermore, previous ${ }^{177} \mathrm{Lu}$-PSMA treatment was negatively associated with PFS in both univariate and multivariate analyses.

Key Words: prostate carcinoma; ${ }^{225} \mathrm{Ac}$; PSMA; radioligand therapy; PSA response

J Nucl Med 2020; 61:62-69

DOI: 10.2967/jnumed.119.229229

$\mathbf{P}$ rostate-specific membrane antigen (PSMA) has been shown to be significantly overexpressed in prostate carcinoma compared with normal and benign hypertrophic prostate tissue $(1,2)$. Furthermore, its overexpression in prostate carcinoma has been shown to be directly related to the tumor Gleason score and disease stage. Of interest, PSMA bears 2 distinct enzyme activities; it can either hydrolyze $N$-acetyl-L-aspartyl-L-glutamate to aspartate and glutamate (NAALDase activity) or release glutamic acid from folate polyglutamate, resulting in the release of folic acid (3). In recent years, modified forms of inhibitors of NAALDase activity that mimic the $\mathrm{N}$-acetyl-L-aspartyl-L-glutamate substrate and that are internalized intracellularly after binding to PSMA have been designed and used as PSMA-targeting agents for prostate carcinoma imaging as well as treatment (1). In terms of treatment, both ${ }^{177}$ Lu-labeled PSMA-617 and PSMA-I\&T have been applied in compassionate-use programs for metastatic castration-resistant prostate carcinoma (mCRPC) patients who have run out of approved treatment options $(4,5)$. However, only $45 \%$ of patients thus treated show a significant decline in prostate-specific antigen (PSA) levels ( $\geq 50 \%$ ), whereas approximately $30 \%$ of patients do not respond at all $(4,5)$.

${ }^{223} \mathrm{Ra}$-dichloride is an approved $\alpha$-emitting radionuclide with survival benefit in patients with predominantly bone metastases of prostate cancer (6). As opposed to $\beta$-emitting isotopes, such as ${ }^{177} \mathrm{Lu}$, that predominantly induce single-strand breaks, $\alpha$-emitting isotopes have significantly higher linear energy transfer (LET) values and thus a greater potential for inducing double-strand breaks and DNA cluster breaks and for cell killing. Their short 
range $(<0.1 \mathrm{~mm})$ may also prove beneficial for reducing toxicity to radiosensitive normal tissues that are invaded by prostate cancer cells, such as normal bone marrow (3). Accordingly, the development of radiolabeled PSMA-targeting inhibitors has recently shifted toward $\alpha$-emitting PSMA-targeting inhibitors. Of the limited $\alpha$-emitting radionuclides that are suitable for clinical application, ${ }^{225} \mathrm{Ac}$ (half-life, $9.9 \mathrm{~d}$ ) and its short-lived daughter radionuclide ${ }^{213} \mathrm{Bi}$ (half-life, $46 \mathrm{~min}$ ) have been most extensively studied $(3,7,8)$. Because of its decay scheme, with 6 daughter products $\left({ }^{221} \mathrm{Fr},{ }^{217} \mathrm{At},{ }^{213} \mathrm{Bi}\right.$, ${ }^{213} \mathrm{Po},{ }^{209} \mathrm{~Pb}$ and ${ }^{209} \mathrm{Tl}$ ) with several $\alpha$ - and $\beta$-decays, ${ }^{225} \mathrm{Ac}$ appears to be promising for clinical applications; preliminary results obtained using PSMA-617 labeled with ${ }^{225} \mathrm{Ac}$ in patients with advanced prostate carcinoma have shown its therapeutic potential $(9,10)$. In a series of 40 advanced prostate carcinoma patients studied by Kratochwil et al., ${ }^{225}$ Ac-PSMA-617 resulted in a PSA decline of more than $50 \%$ in $63 \%$ of patients, with a median duration of tumor control of 9 mo (vs. a median duration of $10 \mathrm{mo}$ for firstline arbiraterone treatment) (9).

Here we report on our experience with a series of 73 castrationresistant prostate carcinoma patients treated with ${ }^{225}$ Ac-PSMA617 , identifying variables predictive for overall survival (OS) and progression-free survival (PFS) after ${ }^{225}$ Ac-PSMA-617 treatment.

\section{MATERIALS AND METHODS}

In this retrospective study, we report our experience on the use of ${ }^{225}$ Ac-PSMA- 617 for the treatment of consecutive patients with histologically proven mCRPC that relapsed after initial therapy with radical prostatectomy, bilateral orchiectomy, external-beam radiotherapy, or prostate brachytherapy and subsequent androgen deprivation therapy with gonadotropin-releasing hormone analogs. Inclusion criteria for treating patients with ${ }^{225}$ Ac-PSMA-617 included a life expectancy of at least $6 \mathrm{mo}$; widespread disease, precluding treatment with radiotherapy with a curative intent; completed chemotherapy or patients' refusal of chemotherapy; and lack of access to second-generation antiandrogen therapy (arbiraterone and enzalutamide). Exclusion criteria were impaired bone marrow function (hemoglobin concentration of $<6 \mathrm{~g} / \mathrm{dL}$, platelet count of $<25 \times 10^{9} / \mathrm{L}$, or white blood cell count of $<3.0 \times 10^{9}$ ); compromised renal function, defined as a glomerular filtration rate of less than $30 \mathrm{~mL} / \mathrm{min} / 1.73 \mathrm{~m}^{2}$ of body surface area; impaired liver function, defined as an albumin concentration of less than $25 \mathrm{~g} / \mathrm{L}$; or uptake of ${ }^{68} \mathrm{Ga}$-PSMA-11 lower than twice the physiologic uptake in the normal liver on PET/CT imaging.

The decision to treat patients with ${ }^{225}$ Ac-PSMA- 617 was made by the local interdisciplinary tumor board. All patients were well informed of the fact that ${ }^{225}$ Ac-PSMA-617 is an experimental treatment agent not approved either in South Africa or elsewhere in the world and of the possible adverse events related to this treatment, especially dry mouth, bone marrow suppression, renal impairment, and the possibility of any other side effects that may be unknown at this time. We obtained a section 21 approval from the South African Health Products Regulatory Authority for the compassionate use of ${ }^{225}$ Ac-PSMA617. All patients to be treated signed a written informed consent form. All procedures performed in this retrospective evaluation were in accordance with the 1964 Declaration of Helsinki and its later amendments or comparable ethics standards.

\section{Patient Preparation}

All patients underwent ${ }^{68} \mathrm{Ga}$-PSMA-11 PET/CT imaging as part of their initial assessment to determine their suitability for ${ }^{225}$ Ac-PSMA617 treatment. Patients were considered suitable for treatment if all identified metastatic lesions demonstrated uptake greater than twice the normal physiologic liver uptake. ${ }^{68} \mathrm{Ga}$-PSMA-11 was synthesized and PET/CT imaging was performed as previously reported $(11,12)$.

\section{Preparation and Administration of ${ }^{225}$ Ac-PSMA-617}

The PSMA-617 precursor (ABX Advanced Biochemical Compounds) was labeled with ${ }^{225} \mathrm{Ac}$ (JRC) in-house and administered to patients as previously reported (10). The initial administration was 8 $\mathrm{MBq}$. Administered activity was deescalated in subsequent cycles to 7,6 , or $4 \mathrm{MBq}$ on the basis of a response to treatment administered earlier as previously described (10). Treatment was repeated every $8 \mathrm{wk}$.

\section{Safety}

All patients were observed for a minimum of $4 \mathrm{~h}$ after ${ }^{225}$ Ac-PSMA617 administration to detect any immediate side effects. Within $2 \mathrm{wk}$ before the first cycle of treatment, all patients underwent determination of their hemoglobin level, leukocyte count, platelet count, glomerular filtration rate, and liver function tests for baseline assessment. Except when clinical situations warranted more frequent follow-up, these blood tests were repeated 2 wk before subsequent cycles of treatment (i.e., every $8 \mathrm{wk}$ ). After completion of the treatment cycles, these blood tests were repeated every $12 \mathrm{wk}$ until disease progression or death. Patients who developed toxicity were monitored until resolution or death. In addition to blood tests, patients reported any observed side effects during treatment or at follow-up. During patients' visits for every cycle of treatment and for follow-up evaluations, each patient was asked about side effects known to occur with PSMA-based radioligand therapy, including dry mouth, dry eyes, dysgeusia, weight loss, anorexia, fatigue, constipation, and dyspepsia. Toxicity was defined according to the Common Terminology Criteria for Adverse Events, version 5.0.

\section{Evaluation of Response to Treatment}

The treatment response was assessed using serial measurements of PSA. PSA was obtained at baseline and subsequently every 4 wk to determine the PSA response to therapy. ${ }^{68} \mathrm{Ga}$-PSMA PET/CT imaging was repeated every $8 \mathrm{wk}$ (before each subsequent cycle of treatment was administered) to guide the activity administered in subsequent treatment cycles as previously described (10). The PSA response and progression were determined using the Prostate Cancer Clinical Trials Working Group 3 criteria, in which a decline in PSA levels of greater than or equal to $50 \%$ was defined as a biochemically significant response (13). Additionally, any change in PSA levels was documented and analyzed. Follow-up ${ }^{68} \mathrm{Ga}$-PSMA-11 PET/CT imaging was used to define the resolution of metastatic lesions initially identified on a baseline scan.

\section{Statistical Analysis}

Statistical analysis was performed using the commercially available software package SPSS, version 25.0 (IBM SPSS). The KolmogorovSmirnov test was used to check the normality of the data distribution. Quantitative variables were compared using a paired Student $t$ test and ANOVA or a Mann-Whitney test and a Kruskal test if their distribution was not normal. The $\chi^{2}$ test and Fisher exact test were used to determine differences in proportions when appropriate.

PFS was calculated from the date of the first ${ }^{225}$ Ac-PSMA-617 treatment. Persistent or recurrent disease at a presenting primary site was scored as local disease failure. PFS took into account all disease events, including local, regional, and distant failures. Patients were followed-up until lost to follow-up or death. OS was defined as the time from the date of the first ${ }^{225}$ Ac-PSMA-617 treatment until death or until the last follow-up. OS took into account all deaths.

For univariate and regression analyses, we dichotomized values according to the median values of the study cohort in the case of continuous variables. We also dichotomized the following clinical covariates: disease stage (IVA vs. IVB), previous radiotherapy, previous 
chemotherapy, the presence of visceral metastases, PSA response of greater than or equal to 50\%, any decline in PSA, and undetectable PSA. PFS and OS were estimated by the Kaplan-Meier method and log-rank testing to examine the predictive value of dichotomized values and other clinical risk factors for local disease control and OS. Multivariate analysis was performed using Cox regression analysis and included, in sequential order of statistical significance, variables that were found to be significant in the univariate analysis followed by the interactive terms.

\section{RESULTS}

\section{Patient Characteristics}

Seventy-three mCRPC patients were included in the present study. The median age of the patient population under study was 69 y (range, 45-85 y). Thirty-seven patients had an Eastern Cooperative Oncology Group (ECOG) score of 0, 23 patients had an ECOG score of 1, 11 patients had an ECOG score of 2, and 2 patients had an ECOG score of 3. Seven patients had isolated lymph node involvement (stage IVA disease); the remaining 66 patients all had bone metastases (stage IVB disease), and 6 of these also had visceral metastases ( 3 patients with liver metastases, 2 with lung metastases, and 1 with both liver and brain metastases). Among the 66 patients with bone metastases, 28 patients had widespread diffuse skeletal metastases in the typical superscan (widespread diffuse skeletal metastases) pattern on ${ }^{68}$ Ga-PSMA-11 PET/CT, including 2 patients with associated visceral metastases (Supplemental Table 1) (supplemental materials are available at http:// jnm.snmjournals.org).

Twenty-seven patients had received previous chemotherapy, 37 had received previous radiation therapy, 1 had received previous arbiraterone and enzalutamide treatment, and 10 had received previous ${ }^{177} \mathrm{Lu}-\mathrm{PSMA}-617$ therapy.

A total number of 210 cycles were administered; 17 patient received 1 cycle, 18 received 2 cycles, 14 received 3 cycles, 11 received 4 cycles, 9 received 5 cycles, 3 received 6 cycles, and 1 received 8 cycles. Patient follow-up ranged from 2 to 22 mo (median, 9 mo). The median number of cycles administered was 3 (range, 1-8 cycles). Additional patient information is shown in Table 1.

After ${ }^{225}$ Ac-PSMA-617 treatment, 51 patients (70\%) had a PSA decline of greater than or equal to $50 \%$, and 60 patients $(82 \%)$ had any decline in PSA (Fig. 1). After ${ }^{225}$ Ac-PSMA-617 treatment, PSMA PET imaging results became negative in 21 patients; that is, avidity was similar to background blood-pool activity (Supplemental Table 2).

\section{Safety}

The administration of ${ }^{225}$ Ac-PSMA-617 was well tolerated. No patient showed any feature of immediate toxicity related to its administration. No patient dropped out of the study on the basis of toxicity or any other reason. The most common toxicity was grade I or II dry mouth, observed in $85 \%$ of patients. No patient had grade III dry mouth, and no patient discontinued treatment because of this side effect. Four patients each had dry eye and dysgeusia in addition to dry mouth. No patient had grade IV bone marrow toxicity. Anemia was the most common manifestation of hematotoxicity and was observed in $37 \%$ of patients (22 patients with grade I or II and 5 patients with grade III). Any grade of renal failure was seen in $32 \%$ of patients (18 patients with grade I or II, 3 patients with grade III, and 2 patients with grade IV). The
TABLE 1

Patient Characteristics

\begin{tabular}{|c|c|}
\hline Characteristic & Value \\
\hline No. of patients included & 73 \\
\hline Median age $(y)$ & 69 \\
\hline$\geq 75$ y old & 29 \\
\hline ECOG score of 0 or 1 & 82 \\
\hline ECOG score of $\geq 2$ & 18 \\
\hline Median PSA level (ng/mL) & 57.2 \\
\hline Median alkaline phosphatase level (IU/L) & 154 \\
\hline Alkaline phosphatase level of $>220 \mathrm{IU} / \mathrm{L}$ & 27 \\
\hline Median hemoglobin level (g/dL) & 11.7 \\
\hline Hemoglobin level of $\leq 10 \mathrm{~g} / \mathrm{dL}$ & 30 \\
\hline Bone metastases & 90 \\
\hline Superscan pattern & 38 \\
\hline \multicolumn{2}{|l|}{ Visceral metastases } \\
\hline Lung & 3 \\
\hline Liver & 5 \\
\hline Brain & 1 \\
\hline \multicolumn{2}{|l|}{ Local therapy to prostate } \\
\hline Prostatectomy & 33 \\
\hline Radiation therapy & 14 \\
\hline No local therapy & 53 \\
\hline \multicolumn{2}{|l|}{ Therapy for castration-resistant disease } \\
\hline Chemotherapy & 37 \\
\hline Abiraterone & 1 \\
\hline Enzalutamide & 1 \\
\hline${ }^{177}$ Lu-PSMA-617 & 14 \\
\hline Estimated median OS (mo) & 18 \\
\hline
\end{tabular}

Data are reported as percentages of patients unless otherwise indicated.

ECOG $=$ Eastern Cooperative Oncology Group.

details of toxicity observed in the treated patients are shown in Table 2.

Side effects were prevalent among patients with a superscan pattern, including xerostomia (25/28); hematologic toxicity in $57 \%$ of patients, half of whom had grade III or IV toxicity; renal toxicity (7/28); weight loss (10/28); and fatigue (14/28).

\section{os}

At the time of data analysis, 13 patients had died, and all deaths seemed to be directly related to the underlying prostate adenocarcinoma. Estimated median OS for all study participants was 18 mo (95\% CI, 16.2-19.9 mo). We found no significant difference in the estimated median OS between the patients who were treated for mCRPC with chemotherapy, second-line hormonal therapy, or ${ }^{177} \mathrm{Lu}$-PSMA-617 and those who were not (7 vs. 9.5 mo, respectively; $P=0.333$ ). Similarly, there was no significant difference in the estimated median OS between the patients who had a superscan pattern and those who did not ( 8.5 vs. 9.0 , respectively; $P=$ 0.330) (Supplemental Table 3 ). In the univariate analysis, the following factors were significantly associated with a favorable 


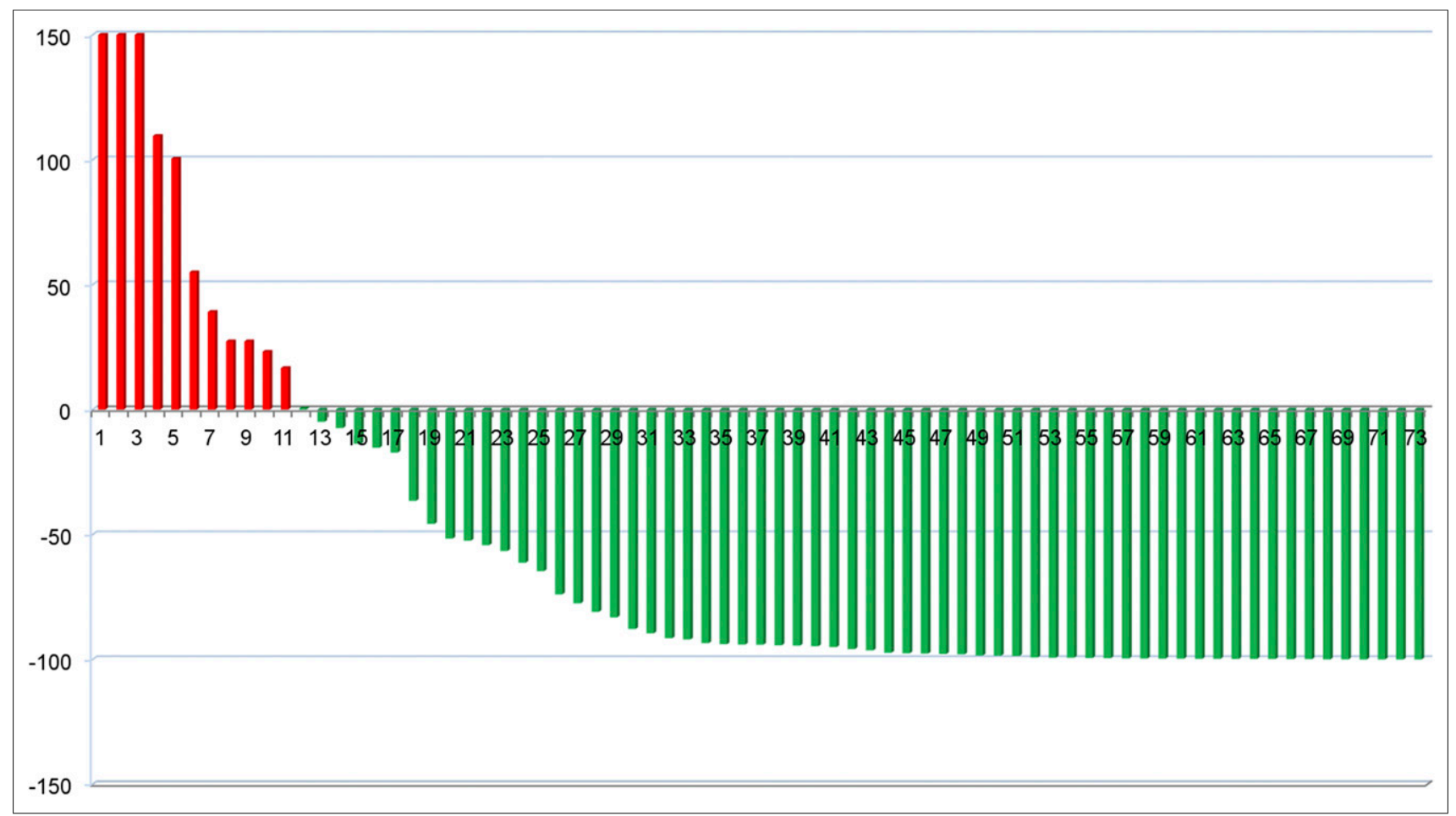

FIGURE 1. Waterfall plot demonstrating percentage change in PSA levels after treatment with ${ }^{225}$ Ac-PSMA-617 in studied patients $(x$-axis $=$ number of patients; $y$-axis $=$ percentage change).

OS: baseline PSA $(P=0.035)$, any PSA decline $(P<0.001)$, PSA decline of greater than or equal to $50 \%(P<0.001)$, hemoglobin level $(P=0.035)$, previous chemotherapy $(P=0.049)$, and

TABLE 2

Toxicity Profiles of 73 Patients Treated with ${ }^{225} \mathrm{Ac}-$ PSMA-617

\begin{tabular}{lccc}
\hline \multicolumn{1}{c}{ Toxic effect } & Grade I or II & Grade III & Grade IV \\
\hline Dry mouth & $62(85)$ & 0 & 0 \\
\hline Dry eyes & $4(5)$ & 0 & 0 \\
\hline Anorexia & $23(32)$ & 0 & 0 \\
\hline Nausea & $15(21)$ & 0 & 0 \\
\hline Vomiting & $4(5)$ & 0 & 0 \\
\hline Constipation & $19(26)$ & 0 & 0 \\
\hline Fatigue & $37(51)$ & 0 & 0 \\
\hline Weight loss & $28(38)$ & 0 & 0 \\
\hline Dyspepsia & $3(4)$ & 0 & 0 \\
\hline Dysgeusia & $4(5)$ & 0 & 0 \\
\hline Anemia & $22(30)$ & $5(7)$ & 0 \\
\hline Leukopenia & $7(10)$ & $2(3)$ & 0 \\
\hline Thrombocytopenia & $6(8)$ & $1(1)$ & 0 \\
\hline Hypoalbuminemia & $14(19)$ & 0 & 0 \\
\hline Renal failure & $18(25)$ & $3(4)$ & $2(3)$ \\
\hline Dysuria & $13(18)$ & 0 & 0 \\
\hline
\end{tabular}

Data are reported as numbers of patients, with percentages of patients in parentheses. previous radiotherapy $(P=0.013)$. When the aforementioned variables were included in the multivariate model with age and Gleason score as covariates, only a PSA decline of greater than or equal to $50 \%$ retained its statistical significance $(P=0.025)$ (Table 3 ; Fig. 2). Estimated OS for patients with a PSA decline of greater than or equal to $50 \%$ was $20.1 \mathrm{mo}$; that for patients with a PSA decline of less than $50 \%$ was 10.5 mo.

\section{PFS}

During follow-up, 23 patients showed disease progression. The estimated PFS for all of the study participants was 15.2 mo (95\% CI, 13.1-17.4 mo). We found no significant difference in the median estimated PFS between patients who had a superscan pattern and those who did not. However, patients' prior treatment for mCRPC had a negative impact on PFS (Supplemental Table 3). In the univariate analysis, the following variables were proven to be significantly related to PFS: baseline PSA $(P=0.020)$, PSA decline of greater than or equal to $50 \%(P<0.001)$, any decline in PSA $(P<0.001)$, undetectable PSA $(P=0.021)$, disease stage $(P=0.048)$, alkaline phosphatase levels $(P=0.040)$, hemoglobin $(P=$ $0.041)$, negative ${ }^{68} \mathrm{Ga}$-PSMA PET scan results $(P=0.001)$, previous radiation therapy $(P=0.002)$, and previous ${ }^{177} \mathrm{Lu}-$ PSMA therapy $(P<0.001)$ (Table 3; Figs. 3A and 3B). When the aforementioned variables were included in the multivariate model with age and Gleason score as covariates, previous treatment with ${ }^{177}$ Lu-PSMA $(P=0.05)$ and a PSA decline of greater than or equal to $50 \%$ retained their statistical significance $(P<0.001)$. Estimated PFS for patients with a PSA decline of greater than or equal to $50 \%$ was $17.9 \mathrm{mo}$; that for patients with a PSA decline of less than $50 \%$ was 6.6 mo. Estimated PFS was much shorter in patients who received 
TABLE 3

Univariate Analysis of Relationship Between Studied Variables and Survival

\begin{tabular}{|c|c|c|}
\hline \multirow[b]{2}{*}{ Variable } & \multicolumn{2}{|c|}{$P^{\star}$ for: } \\
\hline & os & PFS \\
\hline Age & 0.994 & 0.394 \\
\hline Gleason score & 0.163 & 0.120 \\
\hline ECOG score & 0.152 & 0.171 \\
\hline Baseline PSA level (ng/mL) & 0.035 & 0.020 \\
\hline PSA decline of $\geq 50 \%$ & $<0.001$ & $<0.001$ \\
\hline Any PSA decline & $<0.001$ & $<0.001$ \\
\hline PSA undetectable & 0.119 & 0.021 \\
\hline Stage IVA or IVB & 0.576 & 0.048 \\
\hline Visceral metastases & 0.806 & 0.147 \\
\hline No. of treatment cycles & 0.409 & 0.166 \\
\hline Alkaline phosphatase level & 0.318 & 0.040 \\
\hline Hemoglobin level & 0.035 & 0.041 \\
\hline Platelet count & 0.478 & 0.698 \\
\hline White blood cell count & 0.358 & 0.442 \\
\hline $\begin{array}{l}\text { Negative }{ }^{68} \mathrm{Ga}-\mathrm{PSMA} \mathrm{PET} \\
\text { results after treatment }\end{array}$ & 0.175 & 0.001 \\
\hline Previous chemotherapy & 0.049 & 0.169 \\
\hline Previous radiotherapy & 0.013 & 0.002 \\
\hline Previous ${ }^{177} \mathrm{Lu}$-PSMA therapy & 0.146 & $<0.001$ \\
\hline
\end{tabular}

${ }^{*}$ Determined by log-rank test. Values in bold are significant. ECOG $=$ Eastern Cooperative Oncology Group.

${ }^{177} \mathrm{Lu}-\mathrm{PSMA}$ therapy for their disease before Ac-PSMA-617 therapy $(5.1 \mathrm{mo}$; 95\% CI, 3.8-6.5 mo) than in patients who did not (16.5 mo; 95\% CI, 14.3-18.7 mo).

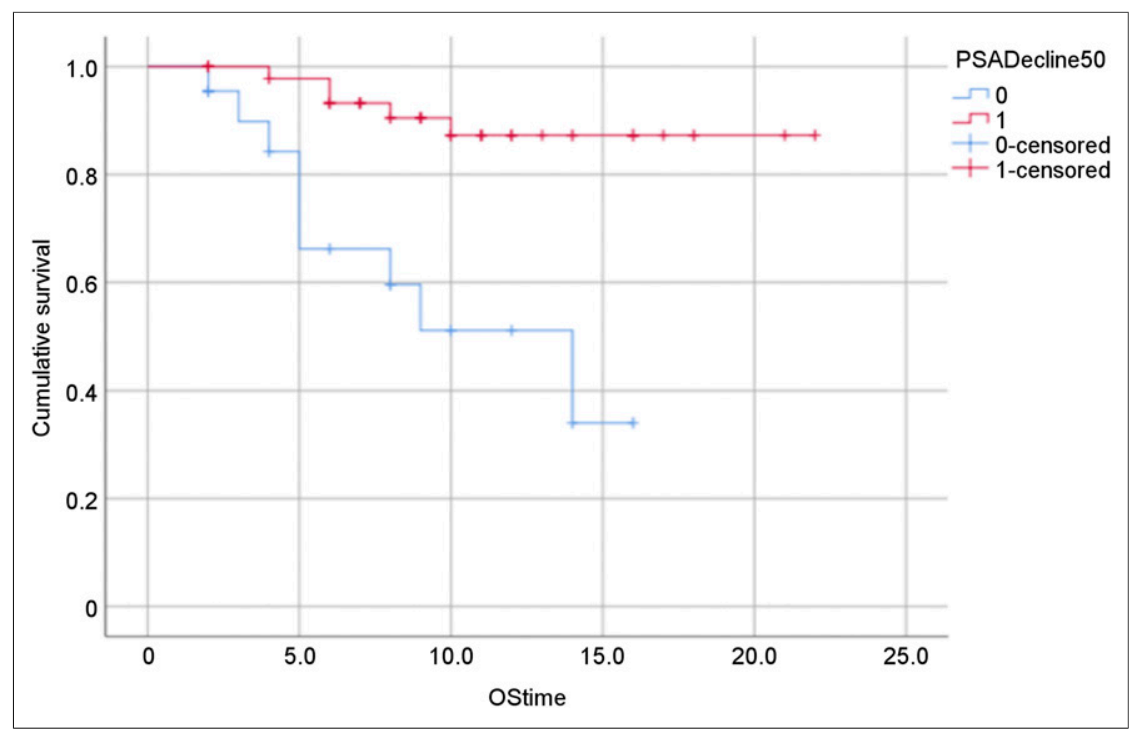

FIGURE 2. Kaplan-Meier plot of OS in months vs. percentage of PSA decline of $\geq 50 \%$ (red curve) and percentage of PSA decline of $<50 \%$ (blue curve).

\section{DISCUSSION}

In this article, we report the response rate, PFS, and OS for a series of 73 mCRPC patients after ${ }^{225}$ Ac-PSMA-617 therapy.

According to the recommendations of the Prostate Cancer Clinical Trials Working Group 3 (13), the response to therapy in mCRPC patients should be assessed on the basis of the PSA response and is commonly defined as a decline in PSA levels of greater than or equal to $50 \%$. In our series, $70 \%$ of patients had a PSA decline of greater than or equal to $50 \%$, which is significantly higher than the response rates reported in the literature for ${ }^{177} \mathrm{Lu}$ PSMA (34\%-59\%) (4,5). This finding might be explained partly by the heterogeneous patient cohorts included in the various studies and, in particular, by the number of treatments exploited before therapy with ${ }^{177} \mathrm{Lu}$ - or ${ }^{225}$ Ac-PSMA-617; these differences make a direct comparison difficult. However, the high response rates observed in the present study and in the study of Kratochwil et al. (9) are probably also due to the significantly higher LET values and thus the anticipated greater potential of $\alpha$-emitting agents than of $\beta$-emitting agents for inducing double-strand breaks and cell killing. In our series, a PSA decline of greater than or equal to $50 \%$ was proven to be the only significant factor associated with a favorable OS in multivariate analyses; the median estimated OS in patients with a PSA decline of greater than or equal to $50 \%$ was $20.1 \mathrm{mo}$, and that in patients with a PSA decline of less than $50 \%$ was $10.5 \mathrm{mo}$. These findings agree with the outcome from the only published phase 2 trial on PSMA-based radioligand therapy, in which Hofman et al. also reported that a PSA decline of greater than or equal to $50 \%$ had a significant survival benefit (14).

To date, to the best of our knowledge, only 4 studies have reported on the factors governing the OS and disease-free survival of mCRPC patients after ${ }^{177} \mathrm{Lu}$-PSMA therapy in multivariate analyses: studies by Bräuer et al. (15), Heck et al. (16), and 2 studies by Ahmadzadefahr et al. (17,18). These studies included 59, 100, 52, and 100 mCRPC patients treated with ${ }^{177} \mathrm{Lu}-\mathrm{PSMA}$, respectively. In none of these studies were PSA levels of greater than or equal to $50 \%$ after the first treatment cycle associated with a significantly longer OS in multivariate analyses. This finding is not at all surprising given the overall lower biochemical response to ${ }^{177} \mathrm{Lu}$-PSMA treatment, with a lower percentage of patients showing a decline in PSA levels of greater than or equal to $50 \%$, resulting in unbalanced groups for univariate analyses and thus lower statistical power. In this regard, the study by Ahmadzadefahr et al. (18) including 100 patients is of interest, because dichotomizing patients according to a lower threshold for the PSA response $(\geq 14 \%)$ yielded 2 more groups for comparison; the PSA response so defined was proven to be associated with a significantly longer median OS (70 wk [ $\sim 16$ mo]; 95\% CI, 39.5100.5 wk) than a PSA decline of less than $50 \%$ (49 wk [ 11 mo]; CI, 30.2-67.8 wk) in both univariate and multivariate analyses.

In contrast to the findings of Ahmadzadefahr et al. (18), the level of serum hemoglobin and the presence of visceral metastases were proven to be not significantly related to OS in multivariate analyses in the present study. 


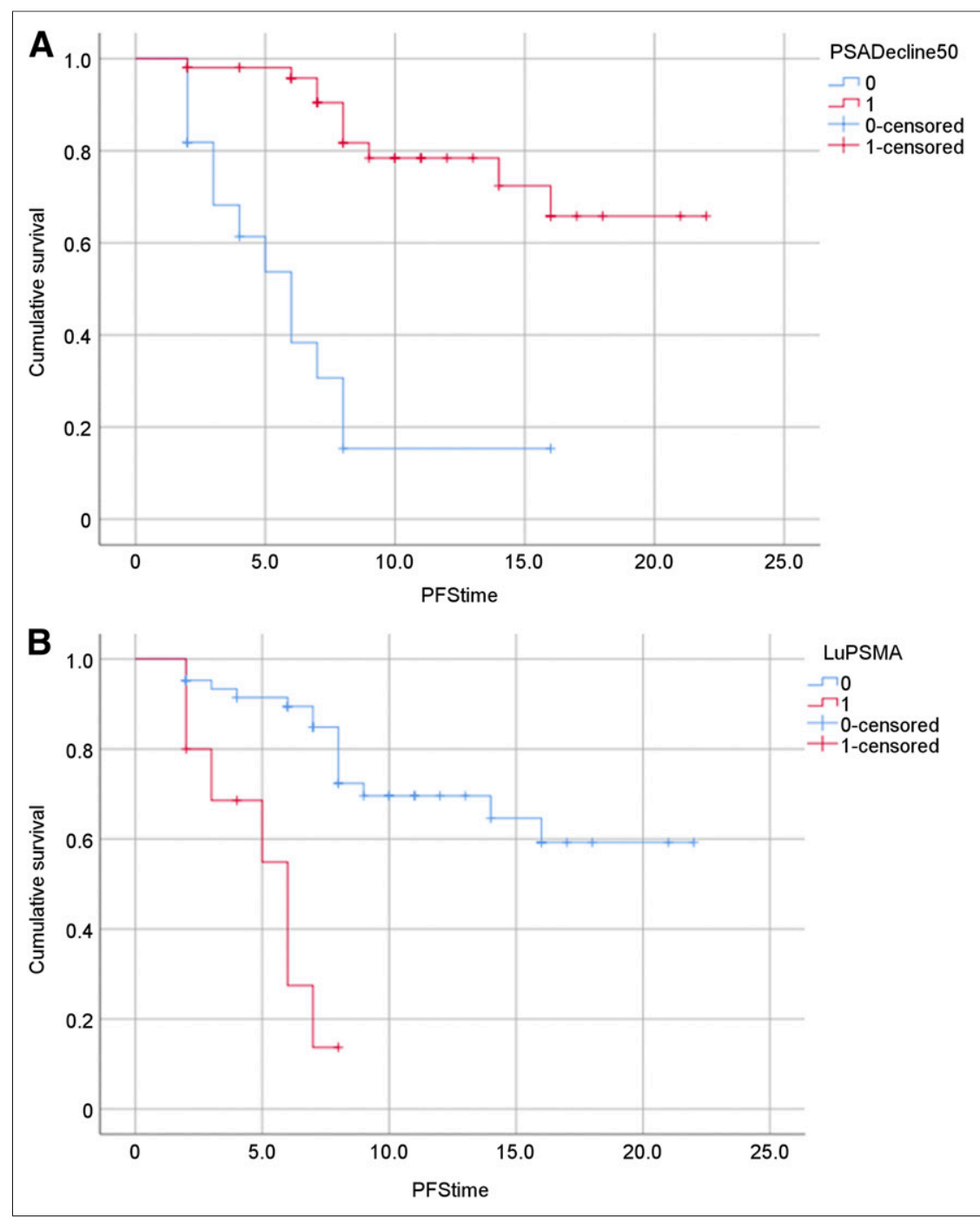

FIGURE 3. (A) Kaplan-Meier plot of PFS in months vs. percentage of PSA decline of $\geq 50 \%$ (red curve) and percentage of PSA decline of $<50 \%$ (blue curve). (B) Kaplan-Meier plot of PFS in months vs. previous pretreatment with ${ }^{177}$ Lu-PSMA-617 (red curve) or no pretreatment (blue curve).

In cancer patients, a low level of hemoglobin is common and has been well documented to impair the quality of life and reduce locoregional disease control conferred by radiation therapy. The reported prognostic importance of low hemoglobin in radiation oncology has been assumed to be related to a reduction in molecular oxygen levels, resulting in attenuation of radiation-induced damage and ultimate cell death $(19,20)$. The oxygen enhancement ratio (the ratio of dose administered under hypoxia to aerated conditions needed to achieve the same biologic effect) has been shown to be 1.0 when LET is greater than $165 \mathrm{keV} / \mu \mathrm{m}$, to vary from 2.0 to 1.3 when LET is between 61 and $110 \mathrm{keV} / \mu \mathrm{m}$, and to increase to 2.4 when LET is reduced to $26 \mathrm{keV} / \mu \mathrm{m}$ (21). Cell death induced by $\alpha$-radiation has also been demonstrated to be independent of cellular oxygenation (22). Accordingly, the significantly higher LET value of ${ }^{225} \mathrm{Ac}(100 \mathrm{keV} / \mu \mathrm{m})$ than of ${ }^{177} \mathrm{Lu}$ $(0.7 \mathrm{keV} / \mu \mathrm{m})$ results in cell killing of ${ }^{225} \mathrm{Ac}$ being significantly less dependent on oxygen and hemoglobin levels than that of ${ }^{177} \mathrm{Lu}$; this fact may partly explain the lack of predictive power of hemoglobin values in multivariate analyses in our series. With regard to visceral metastases, the fact that our series included only a limited number of patients with visceral metastases might have affected statistical power.

The results obtained with large cohorts of mCRPC patients treated with first-line chemotherapy and arbiraterone acetate showed baseline PSA to be predictive for OS in univariate and multivariate analyses. Using data from a phase 3 trial of 1,050 patients (Cancer and Leukemia Group B CALGB-90401), Halabi et al. (23) defined an updated prognostic model for predicting OS after first-line chemotherapy for castration-resistant prostate cancer. Nine parameters were proven to be significantly associated with OS, including PSA levels before treatment (23). Likewise, in a recent observational study reporting on data collected from 481 chemotherapy-naïve patients recruited in 3 European countries and treated with arbiraterone acetate plus corticoids of choice, baseline PSA levels were proven to be 1 of the 6 factors associated with $\mathrm{OS}$ in multivariate analyses (24). In our series, both baseline PSA levels and alkaline phosphatase levels were proven to be significantly related to OS in univariate analyses but not in multivariate analyses, suggesting that for ${ }^{225} \mathrm{Ac}$ PSMA-617 treatment, it is not the number of lesions present but their intrinsic sensitivity to radiation that governs the response to therapy and thus OS.

We additionally assessed the prognostic value of various variables for PFS and found previous treatment with ${ }^{177} \mathrm{Lu}-\mathrm{PSMA}$ to be significantly associated with PFS in univariate and multivariate analyses, suggesting that previous ${ }^{177} \mathrm{Lu}-\mathrm{PSMA}$ treatment may have induced an increased resistance to radiation. Various studies have demonstrated that radioresistance of cancer is due to the existence of intrinsic cancer stem cells (CSCs), termed prostaspheres in prostate carcinoma, that represent a small radioresistant cell subpopulation (25). Compared with adherent prostate cancer cells (non-stem cells), prostaspheres were shown to exhibit a higher level of expression of DNA repair proteins after exposure to ionizing radiation; these proteins efficiently repair radiation-induced DNA injury, conferring a survival benefit (26). Also, non-stem cell prostate cancer cells were shown to be able to differentiate into CSCs via a process termed epithelial-mesenchymal transition (EMT) (25). Like CSCs, prostate cancer cells that have obtained an EMT phenotype have been proven to be more resistant to radiation therapy (27). Finally, in a study by Wang et al., the proportion of prostate cancer stem cell-like cells in a human prostate cancer cell culture was shown to increase significantly after exposure to radiation; this finding suggested that radiation may eliminate radiosensitive adult cancer cells in the culture, perhaps by inducing apoptosis, resulting in the enrichment of radioresistant CSCs (28). Future studies investigating 
the underlying pathways that drive radioresistance in prostaspheres and EMT-transformed prostate cancer cells may lead to the development of treatment approaches that block the generation of radioresistance, thereby potentially improving outcomes after ${ }^{177}$ Lu-PSMA-617, ${ }^{177}$ Lu-PSMA-I\&T, and ${ }^{225}$ Ac-PSMA-617 treatments.

${ }^{225}$ Ac-PSMA-617 treatment was associated with tolerable side effects. Most of our patients experienced dry mouth, which was commonly seen after the first cycle of treatment. This was not severe enough to warrant treatment discontinuation in any patients. In contrast, Kratochwil et al. reported that 4 of 40 patients discontinued treatment because of intolerable xerostomia (9). We routinely titrate the administered activity of the therapy agent against residual disease, as seen on ${ }^{68} \mathrm{Ga}$-PSMA PET/CT, which is done before every cycle of treatment. All patients are initially treated with $8 \mathrm{MBq}$ for the first cycle of treatment, and the administered activity of the treatment agent is reduced in subsequent treatment cycles on the basis of the residual disease seen. This deescalation of treatment might have been responsible for the lesser severity of dry mouth seen in our series. Dry mouth occurred with other gastrointestinal side effects, including dysgeusia, constipation, and weight loss. Hematotoxicity seen in our cohort was transient, and no patient had grade IV hematotoxicity. We observed grade I or II anemia in 22 patients, leukopenia in 7 patients, and thrombocytopenia in 6 patients. Although these bone marrow toxicities were transient, their incidence was relatively high, as ${ }^{225} \mathrm{Ac}$ is expected to spare the functional bone marrow because of its short range. We believe that the incidence of marrow toxicity seen in the present study was related to the preponderance of skeletal metastases in 66 patients, including 28 patients with a superscan pattern on ${ }^{68} \mathrm{Ga}-\mathrm{PSMA}-11 \mathrm{PET} / \mathrm{CT}$. Eight cases of grade III hematotoxicity were seen in 5 patients ( 5 with grade III anemia, 2 with grade III leukopenia, and 1 with grade III thrombocytopenia), all of whom had, at the baseline assessment, impaired bone marrow function resulting from toxicity from their previous treatments. Grade III or IV renal failure was seen in 5 patients, all of whom had renal failure (grade II) before ${ }^{225}$ Ac-PSMA-617 therapy.

The main limitations of the present study were its single center setup and its retrospective design. In line with the ongoing phase 3 randomized VISION study, which will compare ${ }^{177}$ Lu-PSMA-617 plus the best standard of care with the best standard of care alone using OS as the primary endpoint, prospective studies assessing the potential benefit of ${ }^{225}$ Ac-PSMA-617 for PFS and OS are mandatory. Compared with some previously reported patient populations treated with PSMA-based radioligand therapy (9), our cohort appeared to be less heavily pretreated. In the present study, patients who were more heavily pretreated had a significantly shorter duration to biochemical progression than patients in whom ${ }^{225}$ Ac-PSMA-617 was introduced earlier in their treatment history. On the whole, being heavily pretreated did not affect OS in our cohort.

\section{CONCLUSION}

In the present study, a PSA decline of greater than or equal to $50 \%$ after treatment with ${ }^{225}$ Ac-PSMA-617 was proven to be significantly associated with OS and PFS in multivariate analyses. Furthermore, previous ${ }^{177} \mathrm{Lu}$-PSMA treatment was negatively associated with PFS in both univariate and multivariate analyses.

\section{DISCLOSURE}

Frederik L. Giesel is a stock holder of Endocyte, Inc. No other potential conflict of interest relevant to this article was reported.

\section{ACKNOWLEDGMENTS}

We are grateful to Endocyte, Inc., for supplying us with PSMA617. We are also grateful to the staff of the Department of Nuclear Medicine, Steve Biko Academic Hospital, University of Pretoria.

\section{KEY POINTS}

QUESTION: What factors are predictive of overall survival and disease-free survival in patients with metastatic castrationresistant prostate carcinoma treated with ${ }^{225} \mathrm{Ac}-\mathrm{PSMA}-617$ therapy?

PERTINENT FINDINGS: In a cohort of 73 patients with castration-resistant prostate carcinoma treated with ${ }^{225} \mathrm{Ac}$-PSMA617 , there was a prostate-specific antigen (PSA) response in $83 \%$ of patients, with $70 \%$ showing a PSA decline of greater than or equal to $50 \%$. Estimated median overall survival and progression-free survival were 18 and $15.2 \mathrm{mo}$, respectively, and a PSA decline of greater than or equal to $50 \%$ was the strongest predictor of overall survival and progression-free survival.

IMPLICATIONS FOR PATIENT CARE: ${ }^{225 A C-P S M A-617 ~ i s ~ a ~}$ viable treatment option for patients who have castration-resistant prostate carcinoma and for whom conventional therapy has failed; the response is durable, and the side effects are tolerable.

\section{REFERENCES}

1. Schwarzenboeck SM, Rauscher I, Bluemel C, et al. PSMA ligands for PET imaging of prostate cancer. J Nucl Med. 2017;58:1545-1552.

2. Afshar-Oromieh A, Babich J, Kratochwil C, et al. The rise of PSMA ligands for diagnosis and therapy of prostate cancer. J Nucl Med. 2016;57(suppl 3):79S89S.

3. Chakravarty R, Siamof C, Dash A, Cai W. Targeted $\alpha$-therapy of prostate cancer using radiolabeled PSMA inhibtors: a game changer in nuclear medicine. Am J Nucl Med Mol Imaging. 2018;8:247-267.

4. Kim YJ, Kim YI. Therapeutic responses and survival effects of ${ }^{177}$ Lu-PSMA-617 radioligand therapy in metastatic castrate-resistant prostate cancer: a metaanalysis. Clin Nucl Med. 2018;43:728-734.

5. Virgolini I, Decristoforo C, Haug A, Fanti S, Uprimny C. Current status of theranostics in prostate cancer. Eur J Nucl Med Mol Imaging. 2018;45:471-495.

6. Parker C, Nilsson S, Heinrich D, et al. Alpha emitter radium-223 and survival in metastatic prostate cancer. N Engl J Med. 2013;369:213-223.

7. Seidl C. Radioimmunotherapy with $\alpha$-particle-emitting radionuclides. Immunotherapy. 2014;6:431-458.

8. Morgenstern A, Apostolidis C, Kratochwil C, Sathekge M, Krolicki L, Bruchertseifer F. An overview of targeted alpha therapy with ${ }^{225}$ Actinium and ${ }^{213}$ Bismuth. Curr Radiopharm. 2018;11:200-208.

9. Kratochwil C, Bruchertseifer F, Rathke H, et al. Targeted $\alpha$-therapy of metastatic castration-resistant prostate cancer with ${ }^{225}$ Ac-PSMA-617: swimmer-plot analysis suggests efficacy regarding duration of tumor control. J Nucl Med. 2018;59:795-802.

10. Sathekge M, Bruchertseifer F, Knoesen O, et al. ${ }^{225}$ Ac-PSMA-617 in chemotherapy-naive patients with advanced prostate cancer: a pilot study. Eur J Nucl Med Mol Imaging. 2019;46:129-138.

11. Ebenhan T, Vorster M, Marjanovic-Painter B, et al. Development of a single vial kit solution for radiolabeling of ${ }^{68} \mathrm{Ga}$-DKFZ-PSMA-11 and its performance in prostate cancer patients. Molecules. 2015;20:14860-14878.

12. Lawal IO, Ankrah AO, Mokgoro NP, Vorster M, Maes A, Sathekge MM. Diagnostic sensitivity of Tc-99m HYNIC PSMA SPECT/CT in prostate carcinoma: a comparative analysis with Ga-68 PSMA PET/CT. Prostate. 2017;77:1205-1212. 
13. Scher HI, Morris M, Stadler W, et al. Trial design and objectives for castrationresistant prostate cancer: updated recommendations from the Prostate Cancer Clinical Trials Working Group 3. J Clin Oncol. 2016;34:1402-1418.

14. Hofman MS, Violet J, Hicks RJ, et al. $\left[{ }^{177} \mathrm{Lu}\right]$-PSMA-617 radionuclide treatment in patients with metastatic castration-resistant prostate cancer (LuPSMA trial): a single-centre, single-arm, phase 2 study. Lancet Oncol. 2018;19:825-833.

15. Bräuer A, Grubert L, Roll W, et al. ${ }^{177}$ Lu-PSMA-617 radioligand therapoy and outcome in patients with metastasized castration-resistant prostate cancer. Eur J Nucl Med Mol Imaging. 2017;44:1663-1670.

16. Heck MM, Tauber R, Schwaiger S, et al. Treatment outcome, toxicity, and predictive factors for radioligand therapy with ${ }^{177} \mathrm{Lu}$-PSMA-I\&T in metastatic castration-resistant prostate cancer. Eur Urol. 2019;75:920-926.

17. Ahmadzadehfar H, Wegen S, Yordonova A, et al. Overall survival and response patterns of castration-resistant metastatic prostate cancer to multiple cycles of radioligand therapy using ${ }^{177}$ Lu-PSMA-617. Eur J Nucl Med Mol Imaging. 2017; 44:1448-1454.

18. Ahmadzadehfar H, Schlolaut S, Fimmers R, et al. Predictors of overall survival in metastatic castration-resistant prostate cancer patients receiving $\left[{ }^{177} \mathrm{Lu}\right] \mathrm{Lu}-$ PSMA-617 radioligand therapy. Oncotarget. 2017;8:103108-103116.

19. Hoff CM. Importance of hemoglobin concentration and its modification for the outcome of head and neck cancer patients treated with radiotherapy. Acta Oncol. 2012;51:419-432.

20. Khan FA, Shukla A, Joshi S. Anaemia and cancer treatment: a conceptual change. Singapore Med J. 2008;49:759-764.
21. Liu LJ. Model of cell response to $\alpha$-particle radiation. https://arxiv.org/pdf/ 1207.1001. Accessed July 15, 2019.

22. Wulbrand C, Seidl C, Gaertner FC, et al. Alpha-particle emitting ${ }^{213}$ Bi-anti-EGFR immunoconjugates eradicate tumor cells independent of oxygenation. PLoS One. 2013;8:e64730.

23. Halabi S, Lin C, Kelly W, et al. Updated prognostic model for predicting overall survival in first-line chemotherapy for patients with metastatic castration resistant prostate cancer. J Clin Oncol. 2014;32:671-677.

24. Boegemann M, Khaksar S, Bera G, et al. Abiraterone acetate plus prednisone for the management of metastatic castration-resistant prostate cancer (mCRPC) without prior use of chemotherapy: report from a large, international, real world retrospective cohort study. BMC Cancer. 2019;19:60.

25. Li F, Kumming Z, Gao L, et al. Radiation induces the generation of cancer stem cells: a novel mechanism for cancer radioresistance. Oncol Lett. 2016;12: 3059-3065.

26. Kim YS, Kang M, Cho Y. Low production of reactive oxygen species and high DNA repair: mechanism of radioresistance of prostate cancer stem cells. Anticancer Res. 2013;33:4469-4474.

27. Chang L, Graham P, Hao J, et al. Emerging roles of radioresistance in prostate cancer metastasis and radiation therapy. Cancer Metastasis Rev. 2014;33:469496.

28. Wang L, Huang X, Zheng X, et al. Enrichment of prostate cancer stem-like cells from human prostate cancer cell lines by culture in serum-free medium and chemoradiotherapy. Int J Biol Sci. 2013;9:472-479. 